\title{
Spatial correlations in compressible granular flows
}

\author{
T. P. C. van Noije and M. H. Ernst \\ Instituut voor Theoretische Fysica, Universiteit Utrecht, Postbus 80006, 3508 TA Utrecht, The Netherlands \\ R. Brito \\ Departamento de Física Aplicada I, Facultad de Ciencias Físicas, Universidad Complutense, 28040 Madrid, Spain
}

(Received 20 January 1998)

\begin{abstract}
The clustering instability in freely evolving granular fluids manifests itself in the density-density correlation function and structure factor. These functions are calculated from fluctuating hydrodynamics. As time increases, the structure factor of density fluctuations develops a maximum, which shifts to smaller wave numbers (growing correlation length). Furthermore, the inclusion of longitudinal velocity fluctuations changes longrange correlations in the flow field qualitatively and extends the validity of the theory for spatial velocity correlations to higher inelasticities. The theoretical predictions agree well with two-dimensional moleculardynamics simulations. [S1063-651X(98)51205-6]
\end{abstract}

PACS number(s): 81.05.Rm, 05.20.Dd, 05.40. $+\mathrm{j}$

In most studies of rapid granular flows, also called the granular gas regime [1], the inelasticity of granular collisions is assumed to be the most important feature that distinguishes these flows from usual liquid or gas flows. The dynamics is modeled by a single inelasticity parameter $\epsilon=1$ $-\alpha^{2}$, where $\alpha$ is the coefficient of normal restitution. As a consequence granular flows can only be maintained in driven systems, where energy is put into the system, e.g., by gravity, shear, or in vibrated layers [1]. Also, much work has been done on the freely evolving granular fluid [2-6], which has been shown to be linearly unstable (onset of clustering instability) with respect to spatial fluctuations in density, $\delta n(\mathbf{r}, t)=n(\mathbf{r}, t)-\langle n\rangle$ [2]. In both driven and undriven granular fluids there is only a weak separation of microscales and macroscales that makes them behave very differently from molecular fluids. Here we will discuss, for undriven granular fluids, the importance of different intermediate intrinsic scales, related to viscosity, heat conductivity, and compressibility, and controlled by the inelasticity.

The most important function that describes the clustering instability is the structure factor $S_{n n}(k, t)$ $=V^{-1}\langle\delta n(\mathbf{k}, t) \delta n(-\mathbf{k}, t)\rangle$, which is the Fourier transform of the density-density correlation function $G_{n n}(r, t)$ $=V^{-1} \int d \mathbf{r}^{\prime}\left\langle\delta n\left(\mathbf{r}+\mathbf{r}^{\prime}, t\right) \delta n\left(\mathbf{r}^{\prime}, t\right)\right\rangle$ of a system initialized in a spatially homogeneous state. A first step toward a theoretical understanding of $S_{n n}(k, t)$ has been given by Deltour and Barrat [4], who have shown that its growth rate is determined by the most unstable small $k$ part of the heat mode spectrum, but a quantitative theory for the magnitude of $S_{n n}(k, t)$ and its $k$ dependence in the full range of hydrodynamic wave numbers is lacking.

Recently an analytic description of the correlation function $G_{\alpha \beta}(\mathbf{r}, t)$ of the components $u_{\alpha}(\mathbf{r}, t)$ of the flow field has been given in Ref. [7], based on fluctuating hydrodynamics and the assumption of incompressible flow $\boldsymbol{\nabla} \cdot \mathbf{u}=0$. This theory yields predictions, including long-range tails $\sim r^{-d}$ in $d$-dimensional fluids, that, for nearly elastic particles ( $\epsilon$ $\$ 0.2$ ), agree well with two-dimensional moleculardynamics simulations up to large distances. As the transverse velocity fluctuations of the incompressible fluid do not couple to the density fluctuations, the theory of Ref. [7] gives no information on $S_{n n}(k, t)$. Here we will extend that theory to the general compressible case, based on the full set of fluctuating hydrodynamic equations, which allows us to calculate fluctuations in density, temperature, and longitudinal flow fields.

The difference between compressible and incompressible flow is best appreciated by considering $S_{\alpha \beta}(\mathbf{k}, t)$ $=V^{-1}\left\langle u_{\alpha}(\mathbf{k}, t) u_{\beta}(-\mathbf{k}, t)\right\rangle$, the Fourier transform of $G_{\alpha \beta}(\mathbf{r}, t)$. Both tensors can be decomposed into two scalar isotropic functions in the following way:

$$
\begin{aligned}
& G_{\alpha \beta}(\mathbf{r}, t)=\hat{r}_{\alpha} \hat{r}_{\beta} G_{\|}(r, t)+\left(\delta_{\alpha \beta}-\hat{r}_{\alpha} \hat{r}_{\beta}\right) G_{\perp}(r, t), \\
& S_{\alpha \beta}(\mathbf{k}, t)=\hat{k}_{\alpha} \hat{k}_{\beta} S_{\|}(k, t)+\left(\delta_{\alpha \beta}-\hat{k}_{\alpha} \hat{k}_{\beta}\right) S_{\perp}(k, t),
\end{aligned}
$$

where carets denote unit vectors. In a system of elastic hard spheres (EHSs) for times larger than the mean free time $t_{0}$, the correlation functions are given by the equilibrium values, i.e., $G_{\alpha \beta}(\mathbf{r}, t)=[T / m n] \delta_{\alpha \beta} \delta(\mathbf{r})$, containing self-correlations only, and $G_{n n}(r, t)=n \delta(\mathbf{r})+n^{2}[g(r)-1]$, where $g(r)$ is the pair distribution function in thermal equilibrium. For convenience we substract self-correlations and introduce the functions $\quad G_{\alpha \beta}^{+}(\mathbf{r}, t) \equiv G_{\alpha \beta}(\mathbf{r}, t)-[T(t) / m n] \delta_{\alpha \beta} \delta(\mathbf{r}) \quad$ and $S_{\alpha \beta}^{+}(\mathbf{k}, t) \equiv S_{\alpha \beta}(\mathbf{k}, t)-[T(t) / m n] \delta_{\alpha \beta}$. Note that $T(t)$ is measured in energy units $\left(k_{B}=1\right)$. The structure factor of transverse velocity fluctuations $S_{\perp}^{+}(k, t)$ was calculated analytically in Ref. [7] and shown to yield a long-range $r^{-d}$ tail in $G_{\perp}(r, t)$ and $G_{\|}(r, t)$ in case the fluctuations in the flow field are incompressible, i.e., $S_{\|}^{+}(k, t)=0$.

In this Rapid Communication the structure factors $S_{\alpha \beta}(\mathbf{k}, t)$ and $S_{n n}(k, t)$, and corresponding spatial correlation functions $G_{\alpha \beta}(\mathbf{r}, t)$ and $G_{n n}(r, t)$, will be calculated and compared with molecular-dynamics simulations of inelastic hard disk systems. We show in particular by explicit calculation that for small inelasticity $(\epsilon \lesssim 0.2) S_{\|}^{+}(k, t)$ essentially vanishes for all wave numbers, except at very small $k$ values $\left(k \lesssim 1 / \xi_{\|}\right)$, where the assumption of incompressible $\mathbf{u}$ fluctuations, made in Ref. [7], breaks down. Consequently, the 
most important role of $S_{\|}^{+}(k, t)$ is to provide an exponential cutoff for the $r^{-d}$ tail at the largest scales $r \geqslant 2 \pi \xi_{\|}$. At larger inelasticities the contributions from $S_{\|}^{+}(k, t)$ modify $G_{\|}(r, t)$ and $G_{\perp}(r, t)$ significantly at all distances.

The hydrodynamic equations for the unforced inelastic hard sphere (IHS) fluid possess an exact solution, the homogeneous cooling state (HCS), with a homogeneous density $n$ and temperature $T(t)$, and vanishing flow field. Temperature is dissipated at a rate $2 \gamma_{0} \omega T$, where $\gamma_{0}=\epsilon / 2 d$, and where the collision frequency $\omega=\Omega_{d} \chi(n) n \sigma^{d-1} \sqrt{T / \pi m}$ is calculated from the Enskog-Boltzmann equation [8] for a dense system of hard disks or spheres $(d=2,3)$. Here $\Omega_{d}$ $=2 \pi^{d / 2} / \Gamma(d / 2)$ is the surface area of a $d$-dimensional unit sphere, $\sigma$ and $m$ are the sphere diameter and mass, both of which are set equal to unity, and $\chi(n)$ is the pair correlation function at contact. For detailed definitions and derivations we refer to Ref. [2]. In the following, we will assume that IHS hydrodynamics can be described by the standard Navier-Stokes equations supplemented by a term $-2 \gamma_{0} \omega[n, T] T$, evaluated in the local homogeneous cooling state, in the equation of change for the temperature field. A possible justification to lowest order in $\epsilon$ can be found in Ref. [9], as well as a discussion of higher-order terms. The pressure $p=n T\left[1+\Omega_{d} \chi(n) n \sigma^{d} / 2 d\right]$, shear viscosity $\eta$, bulk viscosity $\zeta$, and heat conductivity $\kappa$ are given by the Enskog theory for EHS with a temperature $T(t)$ still depending explicitly on time to account for the homogeneous cooling [7]. The equations of change for the mesoscopic fields are obtained from the hydrodynamic equations by adding fluctuating terms to the pressure tensor and heat flow, denoted by $\hat{\boldsymbol{\Pi}}$ and $\hat{\mathbf{J}}$, respectively. They are characterized by Gaussian white noise, local in space. Their strength is determined by the standard fluctuation-dissipation theorem and related to transport coefficients [10] that depend on $T(t)$ here.

We are interested in the buildup of correlations between spatial fluctuations in a system that is prepared in a homogeneous state at an initial temperature $T_{0}$ and that reaches the HCS within a few mean free times $t_{0}=1 / \omega\left[n, T_{0}\right]$. Therefore, we can linearize around a homogeneous density $n$ and a temperature $T(t)=T_{0} /\left[1+\gamma_{0} t / t_{0}\right]^{2}$, and a vanishing flow field. At this point it is convenient to make the change of variables $d \tau=\omega[n, T(t)] d t$, where $\tau$ is the average number of collisions a particle has suffered within a time $t$; $\delta n(\mathbf{r}, t)=n \delta \nu(\mathbf{r}, \tau), \quad \mathbf{u}(\mathbf{r}, t)=\sqrt{T(t)} \mathbf{w}(\mathbf{r}, \tau), \quad \delta T(\mathbf{r}, t)$ $=T(t) \delta \theta(\mathbf{r}, \tau), \quad \hat{\mathbf{\Pi}}(\mathbf{r}, t)=n T(t) \hat{\boldsymbol{\pi}}(\mathbf{r}, \tau), \quad$ and $\quad \hat{\mathbf{J}}(\mathbf{r}, t)$ $=n T^{3 / 2}(t) \hat{\mathbf{j}}(\mathbf{r}, \tau)$. In these new variables the noise strengths of the reduced fluctuating pressure tensor $\hat{\boldsymbol{\pi}}$ and heat flow $\hat{\mathbf{j}}$ are time independent, and the equations of change for the mesoscopic Fourier modes $\delta \nu(\mathbf{k}, \tau), \mathbf{w}(\mathbf{k}, \tau)$, and $\delta \theta(\mathbf{k}, \tau)$ become ordinary differential equations with time independent coefficients (valid for $k l_{0} \lesssim 1$ where $l_{0}$ $=\sqrt{2 T(t)} / \omega[n, T(t)]$ is the time independent mean free path)

$$
\frac{\partial \delta \nu}{\partial \tau}=-\frac{i k l_{0}}{\sqrt{2}} w_{l}
$$

$$
\begin{gathered}
\frac{\partial w_{\perp \alpha}}{\partial \tau}=\gamma_{0}\left(1-k^{2} \xi_{\perp}^{2}\right) w_{\perp \alpha}-\frac{i k l_{0}}{\sqrt{2}} \hat{\pi}_{\alpha l}, \\
\frac{\partial w_{l}}{\partial \tau}=\gamma_{0}\left(1-k^{2} \xi_{l}^{2}\right) w_{l}-\frac{i k l_{0}}{\sqrt{2}}\left(\frac{p}{n T}\right) \delta \theta \\
-\frac{i k l_{0}}{\sqrt{2}}\left(\frac{1}{n T \chi_{T}}\right) \delta \nu-\frac{i k l_{0}}{\sqrt{2}} \hat{\pi}_{l l}, \\
\frac{\partial \delta \theta}{\partial \tau}=-\gamma_{0}\left(1+k^{2} \xi_{T}^{2}\right) \delta \theta-\frac{i k l_{0}}{\sqrt{2}}\left(\frac{2 p}{d n T}\right) w_{l} \\
-2 \gamma_{0}\left(1+\frac{n}{\chi} \frac{\partial \chi}{\partial n}\right) \delta \nu-\frac{i k l_{0}}{\sqrt{2}}\left(\frac{2}{d}\right) \hat{\jmath}_{l} .
\end{gathered}
$$

Here we have introduced the time independent correlation lengths $\xi_{\perp}, \xi_{l}$, and $\xi_{T}$, defined by $\xi_{\perp}^{2}=\nu / \omega \gamma_{0}$ with $\nu$ $=\eta / m n$, the kinematic viscosity, $\xi_{l}^{2}=[2 \nu(d-1) / d$ $+\zeta / m n] / \omega \gamma_{0}$ and $\xi_{T}^{2}=2 \kappa / d n \omega \gamma_{0}$, and the isothermal compressibility $\chi_{T}=(\partial n / \partial p)_{T} / n$. The subscript $\alpha$ in the equation for $\mathbf{w}_{\perp}$ refers to any of the $(d-1)$ directions perpendicular to $\mathbf{k}$, and the subscript $l$ denotes the longitudinal direction along $\mathbf{k}$.

Since the transverse velocity $\mathbf{w}_{\perp}$ is decoupled from the other modes, its structure factor $S_{\perp}(k, t)$ $=\left\langle u_{\perp \alpha}(\mathbf{k}, t) u_{\perp \alpha}(-\mathbf{k}, t)\right\rangle / V$ can be obtained in the analytic form [7], which is valid for $k l_{0} \lesssim 1$ :

$$
S_{\perp}(k, t)=\frac{T(t)}{n}\left\{1+\frac{\exp \left[2 \gamma_{0} \tau\left(1-k^{2} \xi_{\perp}^{2}\right)\right]-1}{1-k^{2} \xi_{\perp}^{2}}\right\} .
$$

The same result has been obtained from a more microscopic approach, using ring kinetic theory [11].

The density, longitudinal velocity, and temperature modes are coupled and their equations of change can be written in matrix representation as

$$
\frac{\partial}{\partial \tau} \boldsymbol{\psi}(\mathbf{k}, \tau)=\mathbf{M}(\mathbf{k}) \boldsymbol{\psi}(\mathbf{k}, \tau)+\hat{\mathbf{f}}(\mathbf{k}, \tau),
$$

where $\psi$ is the column vector with components $\psi_{1}=\delta \nu$, $\psi_{2}=w_{l}$, and $\psi_{3}=\delta \theta$, and the hydrodynamic matrix $\mathbf{M}$ and the noise vector $\hat{\mathbf{f}}$ are given by Eqs. (2). Note that the elements $M_{31}(\mathbf{k})$ and $M_{33}(\mathbf{k})$, entering the temperature equation, depend on the energy dissipation term. In this notation the equal time correlations obey the equation of change

$$
\begin{aligned}
\frac{\partial}{\partial \tau}\langle & \left.\psi_{\alpha}(\mathbf{k}, \tau) \psi_{\beta}(-\mathbf{k}, \tau)\right\rangle \\
= & M_{\alpha \gamma}(\mathbf{k})\left\langle\psi_{\gamma}(\mathbf{k}, \tau) \psi_{\beta}(-\mathbf{k}, \tau)\right\rangle \\
& +M_{\beta \gamma}(-\mathbf{k})\left\langle\psi_{\alpha}(\mathbf{k}, \tau) \psi_{\gamma}(-\mathbf{k}, \tau)\right\rangle+C_{\alpha \beta}(k),
\end{aligned}
$$

where $\alpha, \beta, \ldots=1,2,3$ label the components $\delta \nu, w_{l}$, and $\delta \theta$. These equations constitute a set of $3 \times 3$ linear ordinary differential equations, of which only six are independent. The matrix of noise strengths $C_{\alpha \beta}(k)$, defined through $\left\langle\hat{f}_{\alpha}(\mathbf{k}, \tau) \hat{f}_{\beta}\left(-\mathbf{k}, \tau^{\prime}\right)\right\rangle=C_{\alpha \beta}(k) \delta\left(\tau-\tau^{\prime}\right)$, has only two 


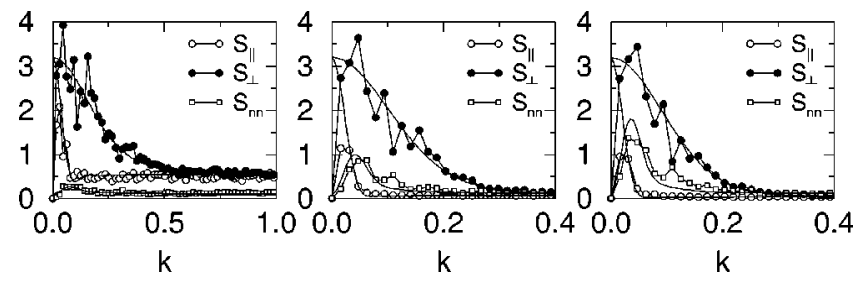

FIG. 1. Theoretical predictions (solid lines) for the structure factors $S_{\perp}(k, t)$ of transverse velocity, $S_{\|}(k, t)$ of longitudinal velocity, and $S_{n n}(k, t)$ of density fluctuations versus $k \sigma$ for $\phi$ $=0.245\left(l_{0} \simeq 0.8\right)$ and $\alpha=0.9$, where $\xi_{\perp}=4$ and $\xi_{\|}=17$ at $\tau$ $=19.4,40$, and 48.4 (from left to right), compared with results from a single molecular-dynamics run of 50000 particles, implying a smallest wave number $k_{\min } \sigma=2 \pi \sigma / L \simeq 0.016$. All structure superimposed on the plateau values presents long-range correlations of dynamic origin; for elastic hard spheres, equilibrium structure in $S_{n n}$ is only present for $k \sigma \gtrsim 2 \pi$.

nonvanishing components, namely $C_{22}=2 V \gamma_{0} k^{2} \xi_{l}^{2} / n$ and $C_{33}=4 V \gamma_{0} k^{2} \xi_{T}^{2} / d n$. We have solved the above set of equations numerically, starting from initial equilibrium correlations, of which the only nonvanishing ones are $\left\langle\psi_{1}(\mathbf{k}, 0) \psi_{1}(-\mathbf{k}, 0)\right\rangle=V T \chi_{T}, \quad\left\langle\psi_{2}(\mathbf{k}, 0) \psi_{2}(-\mathbf{k}, 0)\right\rangle=V / n$, and $\left\langle\psi_{3}(\mathbf{k}, 0) \psi_{3}(-\mathbf{k}, 0)\right\rangle=2 V / d n$ (for $\left.k \neq 0\right)$. The most important results with respect to Ref. [7] are the structure factors $S_{n n}(k, t)$ and $S_{\|}(k, t)$, and the correlation function $G_{n n}(r, t)$. In Fig. 1, we show the results for these structure factors, including $S_{\perp}(k, t)$, for a system with area fraction $\phi=0.245$ and $\alpha=0.9$ together with the results from a molecular-dynamics simulation of 50000 inelastic hard disks. We observe that $S_{\|}(k \rightarrow 0, t)=S_{\perp}(k \rightarrow 0, t)$, implying, for large distances, an asymptotic behavior $G_{\alpha \beta}(\mathbf{r}, t)$ $\sim S_{\perp}(k \rightarrow 0, t) \delta_{\alpha \beta} \delta(\mathbf{r})$, and thus the absence of algebraic long-range correlations on the largest scales $\left(r \gg 2 \pi \xi_{\|}\right)$. Therefore, we can already conclude that the asymptotic behavior of $G_{\perp}(r, t)$ and $G_{\|}(r, t)$ cannot be $r^{-d}$. Instead, the $r^{-d}$ tail obtained in Ref. [7] describes intermediate behavior that is exponentially cut off at a distance determined by the width of $S_{\|}^{+}(k, t)$. This width can be estimated from the eigenvalues of the hydrodynamic matrix, more precisely from the dispersion relation of the "heat mode" [3], which is a pure longitudinal velocity $w_{l}$ for $k \rightarrow 0$. To second order in $k$ its dispersion relation is given by $z_{H}(k)=\gamma_{0}\left(1-k^{2} \xi_{\|}^{2}\right)$ with

$$
\xi_{\|}^{2}=\xi_{l}^{2}+\frac{l_{0}^{2}}{2 \gamma_{0}^{2}}\left[\frac{1}{n T \chi_{T}}-\frac{p}{n T}\left(1+\frac{n}{\chi} \frac{\partial \chi}{\partial n}-\frac{p}{d n T}\right)\right] .
$$

Note that $\xi_{\|} \sim 1 / \epsilon$ for small inelasticity, whereas $\xi_{\perp} \sim \xi_{l}$ $\sim \xi_{T} \sim 1 / \sqrt{\epsilon}$. To a good approximation $S_{\|}(k, t)$ for small wave numbers is given by expression (3) with $\xi_{\perp}$ replaced by $\xi_{\|}$. This approximation is excellent up to wave numbers where the exact numerical result for $S_{\|}(k, t)$ shows a little dip (see Fig. 1, $\tau=19.4, k \simeq 0.1$ ). At about the same wave number the structure factor $S_{n n}(k, t)$ reaches its maximal value, which grows in time. The exact position of this maximum shifts in time to smaller wave numbers corresponding to a growing correlation length. This can be explained by the following argument: for $k \rightarrow 0$ density fluctuations $\delta n(\mathbf{k}, t)$ are decoupled from the heat mode and we expect that $S_{n n}(k \rightarrow 0, t)$ remains at its initial equilibrium value; at small, but nonvanishing $k, \delta n(\mathbf{k}, t)$ couples in $\mathcal{O}(k)$ to the unstable heat mode and the maximum of $k^{2} \exp \left[2 z_{H}(k) \tau\right]$ shifts in time to smaller wave numbers.

The estimate for $S_{n n}(k, t) \simeq S_{n n}(k, 0) \exp \left[2 z_{H}(k) \tau\right]$, used in Ref. [4], differs in two aspects from our predictions: (i) it neglects the wave number dependence of the coupling of density fluctuations to the heat mode, this gives for $S_{n n}(k, t)$, a decreasing function of $k$, and therefore cannot explain the growing correlation length; (ii) it neglects the fluctuating parts of the pressure tensor and heat flow, and thus does not give a quantitative prediction for $S_{n n}(k, t)$. Unfortunately, seven out of the eight sets of data points shown in Fig. 9 of Ref. [4] are in the crossover or nonlinear time regime, which is estimated in Ref. [7] to occur at $\tau_{\mathrm{cr}} \simeq 65$ for $\alpha=0.9$ and $\phi=0.4$ and where our linear theory breaks down.

Using the above approximation for $S_{\|}(k, t)$, the structure factor $S_{\alpha \beta}^{+}(\mathbf{k}, t)$ can be written as

$$
\begin{aligned}
S_{\alpha \beta}^{+}(\mathbf{k}, t) \approx & \frac{T(t)}{n} \int_{0}^{s} d s^{\prime} \exp \left(s^{\prime}\right)\left[\hat{k}_{\alpha} \hat{k}_{\beta} \exp \left(-s^{\prime} k^{2} \xi_{\|}^{2}\right)\right. \\
& \left.+\left(\delta_{\alpha \beta}-\hat{k}_{\alpha} \hat{k}_{\beta}\right) \exp \left(-s^{\prime} k^{2} \xi_{\perp}^{2}\right)\right],
\end{aligned}
$$

where $s=2 \gamma_{0} \tau$. If the system is thermodynamically large $\left(L \gg 2 \pi \xi_{\|}\right), G_{\|}^{+}(r, t)$ and $G_{\perp}^{+}(r, t)$ can be obtained by performing integrals over $\mathbf{k}$ space and are expressed as integrals over simple functions. Here we only quote the results for $d$ $=2$. Using

$$
\int \frac{d \mathbf{q}}{(2 \pi)^{2}} \sin ^{2} \theta e^{i \mathbf{q} \cdot \mathbf{x}-s q^{2}}=\frac{1}{2 \pi x^{2}}\left[1-\exp \left(-x^{2} / 4 s\right)\right],
$$

where $\cos \theta=\hat{\mathbf{q}} \cdot \hat{\mathbf{x}}$, we obtain

$$
\begin{aligned}
G_{\lambda}^{+}(r, t) \approx & \frac{T(t)}{n}\left\{\frac{1}{4 \pi \xi_{\lambda}^{2}} \int_{0}^{s} d s^{\prime}\right. \\
& \times \frac{\exp \left(s^{\prime}-x_{\lambda}^{2} / 4 s^{\prime}\right)}{s^{\prime}}+\frac{\sigma_{\lambda}}{2 \pi r^{2}} \int_{0}^{s} d s^{\prime} e^{s^{\prime}} \\
& \left.\times\left[\exp \left(-\frac{x_{\|}^{2}}{4 s^{\prime}}\right)-\exp \left(-\frac{x_{\perp}^{2}}{4 s^{\prime}}\right)\right]\right\},
\end{aligned}
$$

for $\lambda=\|, \perp$, where $x_{\lambda}=r / \xi_{\lambda}, \sigma_{\|}=1$, and $\sigma_{\perp}=-1$. The approximation of incompressible fluid flow of Ref. [7] is obtained in the limit $\xi_{\|} \rightarrow \infty$. At finite $\epsilon$, Eqs. (9) describe exponentially decaying functions at distances $r \geqslant 2 \pi \xi_{\|}$. Moreover, upon increasing the inelasticity the minimum in $G_{\perp}(r, t)$ becomes less deep and vanishes if $\xi_{\|}=\xi_{\perp}$.

The predicted spatial velocity correlations $G_{\|}(r, t)$ and $G_{\perp}(r, t)$ have been obtained by performing inverse Bessel transformations on the numerical results for $S_{\|}(k, t)$ and $S_{\perp}(k, t)$. The result for $G_{\|}(r, t)$, corresponding to Fig. 1, includes an intermediate $r^{-2}$ tail, as is shown in Fig. 2(a). Figure 2(b) shows the corresponding spatial density correlation $G_{n n}(r, t)$ obtained numerically from $S_{n n}(k, t)$. It confirms that the present theory correctly predicts the buildup of density correlations, including a negative correlation centered around a distance that grows in time as $\sqrt{\tau}$. 
(a)

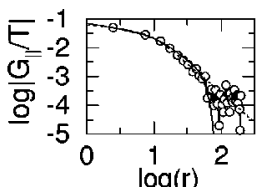

(b)

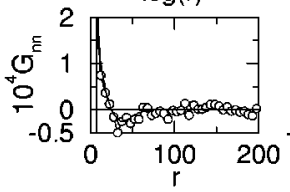

(c)

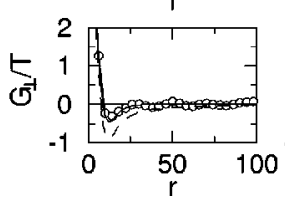

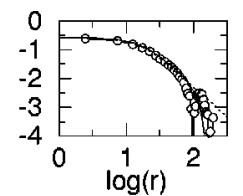
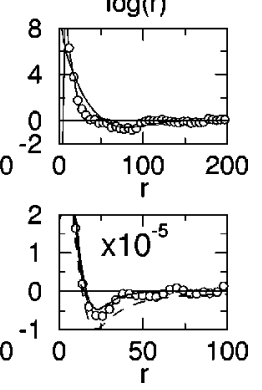

FIG. 2. (a) Longitudinal flow field correlation $\log _{10}\left[\left|G_{\|}\right| / T\right]$ versus $\log _{10} r$. (b) Density-density correlation $10^{4} G_{n n}$ versus $r$; the same parameters $\alpha, \phi, \tau$ as in Fig. 1 are used for (a) and (b); (c) $\mathcal{N} G_{\perp} / T$ versus $r$ for $\phi=0.4\left(l_{0} \simeq 0.34\right), \alpha=0.6 \quad\left(\xi_{\perp}=1.46, \quad \xi_{\|}\right.$ $=3.8$ ) at $\tau=20,40$, and 60 (from left to right) with $\mathcal{N}=1,10^{-2}$, and $10^{-5}$, respectively; in (a) and (c) the solid (dashed) line is the prediction from compressible (incompressible) fluctuating hydrodynamics.

At small inelasticity $(\epsilon \lesssim 0.2)$ the functions $G_{\|}(r, t)$ and $G_{\perp}(r, t)$, calculated here from the full set of hydrodynamic equations, differ for $r \lesssim 2 \pi \xi_{\|}$only slightly from the results for incompressible flow fields (see the discussion in Ref. [7]). However, the algebraic tails $\sim r^{-d}$ in $G_{\|}(r, t)$ and $G_{\perp}(r, t)$, derived in Ref. [7] for $r \geqslant 2 \pi \xi_{\perp}$, are exponentially

cut off for $r \gtrsim 2 \pi \xi_{\|}$, as implied by Eq. (9). As the correlation lengths $\xi_{\perp} \sim 1 / \sqrt{\epsilon}$ and $\xi_{\|} \sim 1 / \epsilon$ are well separated for small $\epsilon$, there is an intermediate range of $r$ values where the algebraic tail $\sim r^{-d}$ in $G_{\|}(r, t)$ can be observed.

At higher inelasticity $\xi_{\|}$and $\xi_{\perp}$ are not well separated and, as a consequence, there does not exist a spatial regime in which the longitudinal fluctuations in the flow field can be neglected and the regime of validity of the incompressible theory of Ref. [7] has shrunk to zero. Figure 2(c) compares results from incompressible and compressible fluctuating hydrodynamics with simulation data for $G_{\perp}(r, t)$ at $\alpha=0.6$ and $\phi=0.4$, and confirms the necessity of including longitudinal velocity fluctuations to calculate the spatial velocity correlations at reasonably large inelasticities. Note that (at any inelasticity) $G_{n n}(r, t)$ can only be calculated from the compressible theory.

Hence, the good quantitative correspondence between our theory (lines) and computer simulations (points in the figures) attests to the correctness of our theory for structure factors $S_{\alpha \beta}(\mathbf{k}, t)$ and $S_{n n}(k, t)$ and spatial correlation functions $G_{\alpha \beta}(\mathbf{r}, t)$ and $G_{n n}(r, t)$ for wave number, position, and time dependence in the relevant hydrodynamic range and for inelasticities $(\alpha \gtrsim 0.6)$ that are not too large.

The authors want to thank J. A. G. Orza for his help in performing the simulations. T.v.N. acknowledges support of the foundation "Fundamenteel Onderzoek der Materie (FOM),' which is financially supported by the Dutch National Science Foundation (NWO). R.B. acknowledges support from DGICYT (Spain) Grant No. PB94-0265.
[1] H. M. Jaeger, S. R. Nagel, and R. P. Behringer, Rev. Mod. Phys. 68, 1259 (1996).

[2] I. Goldhirsch and G. Zanetti, Phys. Rev. Lett. 70, 1619 (1993); I. Goldhirsch, M-L. Tan, and G. Zanetti, J. Sci. Comput. 8, 1 (1993).

[3] S. McNamara, Phys. Fluids A 5, 3056 (1993); S. McNamara and W. R. Young, Phys. Rev. E 53, 5089 (1996).

[4] P. Deltour and J.-L. Barrat, J. Phys. (France) I 7, 137 (1997).

[5] S. E. Esipov and T. Pöschel, J. Stat. Phys. 86, 1385 (1997).

[6] J. J. Brey, F. Moreno, and J. W. Dufty, Phys. Rev. E 54, 445 (1996); J. J. Brey, M. J. Ruiz-Montero, and D. Cubero, ibid.
54, 3664 (1996).

[7] T. P. C. van Noije, M. H. Ernst, R. Brito, and J. A. G. Orza, Phys. Rev. Lett. 79, 411 (1997).

[8] S. Chapman and T. G. Cowling, The Mathematical Theory of Non-uniform Gases (Cambridge University Press, Cambridge, England, 1970).

[9] N. Sela and I. Goldhirsch, J. Fluid Mech. (to be published).

[10] L. Landau and E. M. Lifshitz, Fluid Mechanics (Pergamon Press, New York, 1959), Chap. 17.

[11] T. P. C. van Noije, M. H. Ernst, and R. Brito, Physica A 251, 266 (1998). 